\title{
MANAGEMENT UNIT FOR RELIABLE POWER SUPPLY STATIONS FOR USE IN TELECOMMUNICATION FACILITIES
}

\section{G. GATINE : MERLIN GERIN, SES deparment}

\section{$\begin{array}{ll}\text { J.P. LEBLANC } & \text { :CNETPAB/STC/ESA } \\ \text { D. MARQUET } & \text { :CNET PAB/STC/ESA }\end{array}$}

\section{SUMMARY:}

Following the general presentation of the system architectures for two types of reliable energy stations, GEODE ${ }^{*}$ and ALFATEL used in telecommunication facilities, we will describe the concept of a centralized management system covering severat energy stations situated at different geographical locations.

The role of the functions of communication with the powe supply station monitoring units (US) will then be described.

This will enable us to define the functions carried out by the management unit, some of which may be operated by remot control by staff on standby duty.

Remote monitoring of processes, statistical studies, inventories, background records, man/machine relationships, maintenance assistance.

These functions are backed up by a PC-type machine, whose configuration will be defined at the end of this document.

N.B.* GEODE : Energy management and equipment optimization

ALFATEL : Reliable power supplies for teleconmunication facilities.

\section{1- INTRODUCTION}

The rapid evolution of the French telecommunications network which aims to encompass 30 million lines over the next decade, has resulted more particularly in the development of power supply systems and their operation. Our operating principles are largely based on remote monitoring and telediagnostic techniques, as well as the man/machine relationships, which have aiready been widely developed locally in the GEODE-type power supply stations, designed for autonomous routing, highpower plants (CAA) and production and operation plants (CPE) and ALFATEL, designed for local network, low-power plants and ALFATEL, desig

As far as the remote monitoring and telediagnostic requirements are concerned, the power availability limitations on the application terminals (the automatic switch) have wide-reaching effect on the level of simplicity of the operations and the high level of operator assistance, in orde to :

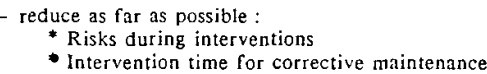

Moreover, the analysis of the ageing and reliability of the subassemblies constituting the station enables optimization of the stock of spare parts for maintenance purposes.

With a view to generalizing the concept of remote monitoring and telediagnostic techniques adapted to these power supply stations, the CNET launched the UGe project (Energy Management Unit) and has entrusted the development and industrialization studies for the installations forming the UG system to MERLIN GERIN.
Following a brief description of the architecture of the GEODE and ALFATEL energy production system, this report will present the architecture and functions enabling the above mentioned objectives to be attained.

\section{2 - A DEFINITION OF THE POIYER SUPPLY STATIONS}

Energy production and distribution is considered as a process, automated by a micro-computer device. The entire process and electronic system can be divided up according to the fou traditional levels of automation of industrial processes. These are: the process level, the command level, the monitoring level, and the management level (cf. Figure 1 and 2).

\section{The process level}

This is the lowest level in the hierarchy. At this level, energy is produced, distributed, and stored by means of specialized processes.

Energy production is ensured by a diesel-motor generator set. This method was chosen because the fuel can be easily stored, This method was chosen because the
thus providing sufficient autonomy.

thus providing sufficient autonomy.
Energy distribution is ensured by the source four-way switches Energy distribution is ensured by the source four-way switches
and remote-controlled circuit-breakers, which distribute the and remote-controlled circuit-breakers, which distribute the
energy, and protect the men and the installation from the energy, and protect the men and the installation
faults liable to appear on the various users" premises.

\section{The command level}

This is the first level of the micro-computer system. Its task is to command the processes described above. The command units (UC) are electronic sub-assemblies, especially adapted to the processes and designed to take into account the aims of availability and maintainability.

\section{The monitoring level}

The maintainability factor is reinforced by this level, as welf as by the management level, and of course the maintenance logistics.

The role of the monitoring units is to detect faults and, wherever possible, 10 determine the original malfunction generating the fault.

\section{The management level}

This is the highest level in the architectural hierarchy. A large number of power supply stations may be connected to the management level via a communications network. which may use the switched public telephone network and/or a packetswitching network.

\section{3 - DEFINITION OF THE POWER SUPPLY SOURCES}

\section{- GEODE Dower supply source}

$\begin{aligned} \text { It is composed of : } & \text { * An EDF mains supply } \\ & \text { A parallel generator set (GE) on the } \\ & \text { EDF supply } \\ & \text { * A low-voltage switcliboard (TBT) } \\ & \text { A short-duration emergency set } \\ & \text { (CONTINU) } \\ \text { consisting of current rectifiers and } & \text { floating batteries }\end{aligned}$




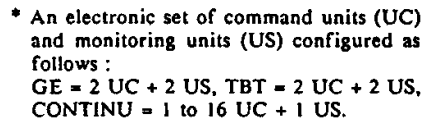

FICURE 1 : Geecral structiore of CEODE

\section{Mrainl cemponds}

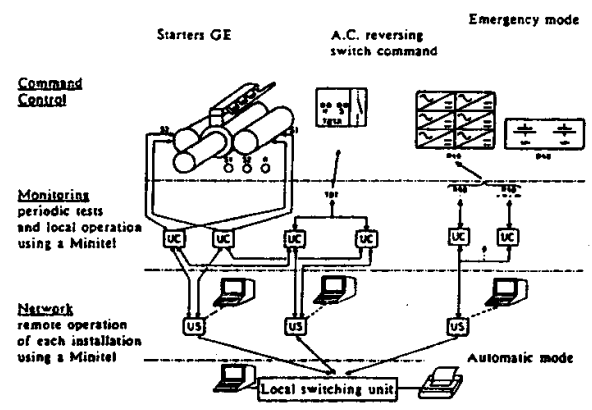

- ALEATEl gower supolv soureg

It is eemposed of: : An EDE supply rack with source iaterchang

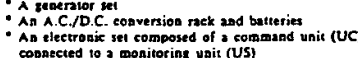

Figure 2: ALFATEL general structure

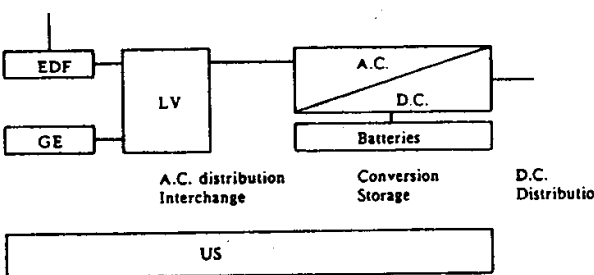

\section{- THE CONCEPT OF POIVER SUPPLY STATION MANAGEMENT}

\section{- Centrallization}

The GEODE- and ALFATEL-type power supply stations supply telecommunication facilities located in the plants (CPE, CAA $C L)$. These plants depend on a logistic organization, centred on the CPE or CPEP production and operating plant. This plant must ensure the management of the means, operations. and preventive and corrective maintenance of associated plants.

The management of the power supply stations located in these centres is carried out by a UGe central installation, which can group together up to 40 high-power GEODE-type stations, and 240 tow-power ALFATEL-type stations.

\section{- Communications (cf. Figure 4)}

The management unit is integrated in an operations computer network ASPECT (Alarm, Security. PEriCommuTation) which interconnects the telephone exchanges.

The communication protocol is based on the standard CCITT $\mathrm{X} 25$ telecommunications architecture.

This network consists of a switching unit (UA) which incorporates :

- the routing node of an X25 network (layers 1 to 3 of the OSI model)

- a step-by-step alarm organization unit for monitoring units (US)

- the display terminal enabling consultation of the monitoring units (US).

All the monitoring units (US) on one site are connected to the switching unit (UA) of that site.

The ASPECT network is composed of tree-data-transmission sub-networks (CPE) interconnected at the UA/CPE or UA/CPEP level (cf. Figure 3 ).

The Uge can communicate with an external unit connected to the TRANSPAC network, via the ASPECT/TRANSPAC bridge, available at the UA/CPE or UA/CPEP level.

The ASPECT network ensures the opening of the transfer of data concerning the systems responsible for monitoring the power supply or building installations.

FIGURE 3 : An example of an ASPECT network connection
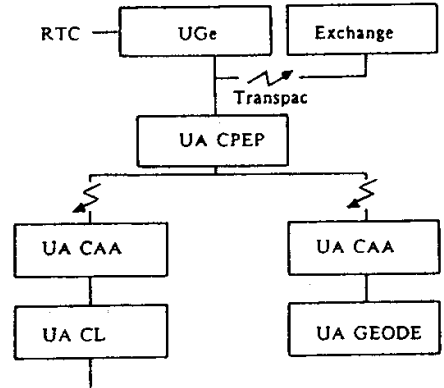

US ALFATEL

\section{5 - UGE CONNECTIONS (cf. Diagram No. 4)}

The UGe can be connected to the following external units :

- The GEODE monitoring units (US) which transfer data, such as the status of the US-linked process system, the log book, the alarm list and the operating parameters to the UGe.

- The ALFATEL monitoring units (US) which transfer system status-type data to the UGe.

- The management computer for the upper level (DO) responsible for receiving the daily files compiled from data from the various monitoring units (US) in order to create background data files and carry out statistical processes. 
- The operator is able to consult the UGe by remote control using a Minitel connected to the switched network.

At a given moment, the UGe can communicate with :

- A UGe operator at the CPEP, via the UGe operato terminal and its printer.

The CPE, CAA and $\mathrm{CL}$ operators, via a Minitel connected to the UGe by the switched telephone network.

The GEODE monitoring units (US) of all the CPE sub-

networks dependent on the UGe-equipped CPEP.

- The ALFATEL monitoring units (US) of all the sub-

networks, dependent on the UGe.

* The management unit located in DO.

\section{6 - UGE FUNCTIONS}

The UGe carries out 6 basic management functions

- Data acquisition from the various station USs.

- Remote monitoring of the stations in the plants

- Consultation of the plants' operating parameters

- Transfer of US files to the centralized management

computer on the upper level.

- Assistance to plant operators

- File storage and updating services

This division of functions is not the only possible division. Naturally, data presentation redundancies, indispensable for the user, also exist :

- between the graphics and the texts

- between the observation levels

- finally, in the data access mode.

\section{6-1 Data_acquisition}

The UGe incorporates the directory of the plants, which contains the list of USs, in chronological order of interrogation, US call-up number and site name.

When periodically consulted by the UGe, the USs in question supply the logbook data. This data is stored for two weeks, thereby allowing an operator to consult it using a local screen, thereby allowing an operator to consult it using a local screen,
or by printing them on a printer connected to the UGe, or by remote control using a Minitel connected to the UGe via the remote
PSTM.

Moreover, this data is transferred to the centralized Moreover, this data is transferred to
management computer for statistical purposes.

The directory can be consulted by the operator, and may be up-dated to allow for the following actions:

- installation of a new US

- removal of a US

- changing a call number

\section{6-2 Remote monitoring of facilities}

This function provides the operator with an instantaneous image of the status of a plant power supply station controlled by the UGe in the form of hierarchical mimic diagrams and their corresponding alarm lists.

The composition of these hierarchical mimic diagrams is based on the site configuration, and the installation and operational parameters supplied by the US of the plant in question.

Each level of the mimic dingram uses the current system statuses and alarm journals of each US to signal events on the mimic diagrams.
One of the major advantages should be the visual transcription of the textual data forming the alarm message, with the two presentations remaining complementary: the mimic diagram provides an overall view of the system status, and the alarm provides an sularm messages enable an analyical understanding of what has complement each other, using their perception of the context.

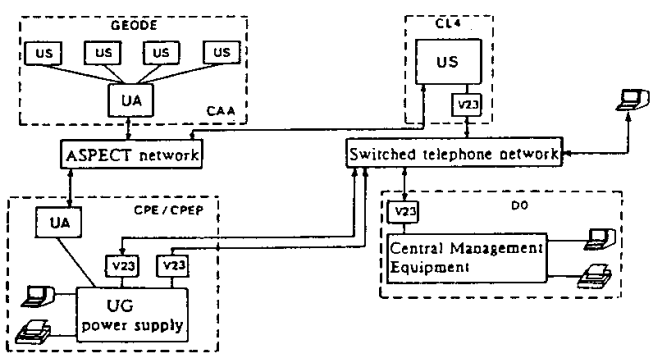

\section{- Consultation procedure (cd. Figure No. Treeing of Mimic} Diagrams)

Following an operator request, the UGe gathers data from all the consulted USs making up the power supply station. The data received enables the UGe to form the general mimic diagram for general consultation purposes, and the process mimic diagram, plus any existing alarm messages (GE, TBT. mimic diagram, plus any existing alarm messages (GE, TBT, visualization of the statuses: process state - in/out, On/Off, application consumption rate, current alarms.

During the GE and CONTINU processes, a circuit level (GE) or rack level (CONTINU) allows access to the sub-assembly indicated by the alarm, thereby efficiently assisting the operator.

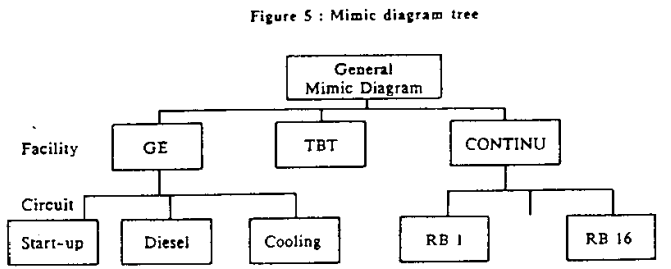

\section{6-3. Maintenance assistance}

The sturdiness and reliability of the facilities reduces the number of interventions in the stations. However, owing to the increasing complexity of the command control and the refined station operating monitoring systems, the operator is all the more in danger of forgetting a particular operation associated with an alarm, thereby necessitating prohibitive repairs resulting in a deiay in the availability of the power supply.

The aim of the maintenance assistance function is to enable the operator to visualize these operations, and so simplify fault location. This function can be accessed from any one of the various mimic diagram consultation levels.

Mimic diagram consultation can be taken up again at the point where the operator left off in order to consult the information sheet. 
If the operator's station has specific characteristics, he can complete the data sheet in the UGe.

6-4 Inventory function

Using this function, the operator can gain access to the specifications of the equipment in each plant.

Using the data contained in the general system statuses, the UGe updates this information table each time it interrogates the USs of the corresponding plant.

\section{7 - SYSTEM CONFIGURATION}

The UGe basic configuration is based on an IBM PC-compatible type microcomputer, and is composed of:

1 printer

- 1 modem, to be connected to a Minitel or Class 4

monitoring unit (US)

- I modem, to be connected to the centralized

management computer

I synchronous coupler, to be connected to the UA of the CPE

\section{8 - FURTHER DEVELOPMENTS}

The functions described at the UGe level have only been included for presentation purposes, but the network and redundancy concepts must be kept in mind. There may be partial overlap with the functions carried out by the USs or the network nodes.

The latter point is certainly important for the operating and mission reliability of the facilities, but it becomes vital for the correct use of data and is accompanied, in the case of a network, by an operator-trassparent functional delocalization. Opening of the network therefore allows for the addition of new resources in a more realistic way than does opening of the software.

Via an operation network, the user will therefore be able to gain access to :

- the actual resources of the USs integrated in the facilities

facilities - the functions induced by the pre-processing and preconcentration provided by the network node in the plant
- finally, the UGe functions, common to several centres, - finally, the UGe functions, common to several centres, both on the present operation.
level of studies and research.

\section{Transiator's note}

EDF Mains supply

CPE Telecommunication mains center

$\mathrm{CL}$ Telecommunication local center

UA Local svitching unit

UC Control unit

US Monitoring unit
UG Management unit

TBT Lov voltage unit

GE Generaling set 